\title{
Inadequate or Incorrect Instructions for Maintenance
}

National Cancer Institute

\section{Source}

National Cancer Institute. Inadequate or Incorrect Instructions for Maintenance. NCI

Thesaurus. Code C139496.

Inadequate or incorrect information in the instructions for maintenance. 